\title{
Gunturk-Altunbasak-Mersereau Alternating Projections Image Demosaicking
}

\author{
Pascal Getreuer \\ CMLA, ENS Cachan (pascal.getreuer@cmla.ens-cachan.fr) \\ Communicated by Antoni Buades Demo edited by Pascal Getreuer
}

\begin{abstract}
The problem of image demosaicking (or demosaicing) is where an image has been captured through a color filter array (CFA), and the goal is to estimate complete color information at every pixel. This IPOL article describes the image demosaicking method proposed by Gunturk, Altunbasak, and Mersereau in "Color Plane Interpolation Using Alternating Projections." Given an initial demosaicking, the method improves the result by alternatingly applying two different projections. One projection copies the green channel's wavelet detail coefficients to the red and blue channels while the other projection constrains the solution to agree with the observed data.
\end{abstract}

\section{Source Code}

ANSI C source code to produce the same results as the demo is accessible on the article web page https://doi.org/10.5201/ipol.2011.g_gapd. This software includes the implementations of algorithms potentially linkable to patents. Various distribution terms apply. Some files are distributed under the terms of the simplified BSD license, some are for scientific and education use only. Future software releases and updates will be posted at http: //dev.ipol.im/ getreuer/code.

\section{Keywords:}

\section{Overview}
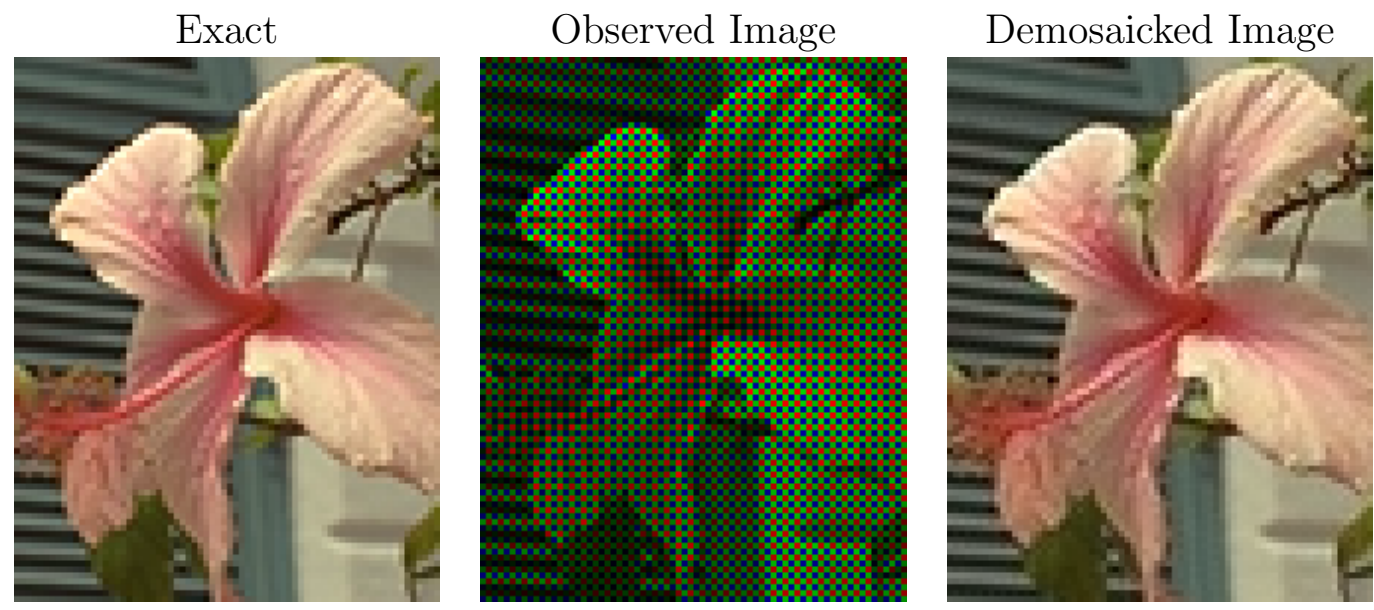
Gunturk, Altunbasak, and Mersereau's demosaicking. The images are enlarged to show individual pixels more clearly.

In the Bayer pattern [1], green pixels cover half the array in a quincunx lattice. The red and blue pixel locations are spaced uniformly every two pixels. The pattern alternates between "red rows" and "blue rows." In a red row the pattern is $R, G, R, G, \ldots$, and in a blue row it is $G, B, G, B, \ldots$

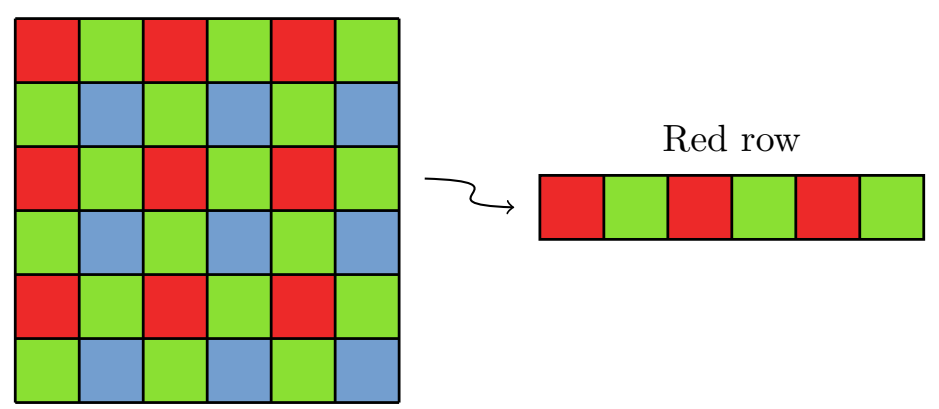

The Bayer pattern.

\section{Redundant Wavelet Transforms}

We first review the redundant wavelet transform (RWT) as this is the central tool used by the algorithm.

In one dimension, the redundant wavelet decomposition of a signal $x(n)$ is computed by convolution with a lowpass filter $h_{0}$ and a highpass filter $h_{1}$,

$$
a=h_{0} * x, \quad d=h_{1} * x .
$$

In the usual wavelet transform, the signals $a$ and $d$ are downsampled. However, for the redundant wavelet transform, they are not. If $x$ is a signal with $N$ samples, then its decomposition $(a, d)$ has $2 N$ samples, hence the representation is redundant. The original signal $x$ is reconstructed from $a$ and $d$ by

$$
x=\left(g_{0} * a\right)+\left(g_{1} * d\right),
$$

where $g_{0}$ and $g_{1}$ are the reconstruction filters. In order for the reconstruction to recover the original signal, the condition on the filters is

$$
\left(g_{0} * h_{0}+g_{1} * h_{1}\right)(n)= \begin{cases}1 & \text { if } n=0, \\ 0 & \text { otherwise }\end{cases}
$$

In two dimensions, a separable redundant wavelet decomposition is performed by applying the onedimensional decomposition along rows and then decomposing the results along columns. This produces four subbands LL, LH, HL, and HH, each having the same size as the original image. For the reverse direction, reconstruction is performed by reconstructing along columns and then reconstructing along rows.

\section{Algorithm}

An initial demosaicking is needed to start the method. This can be done for example with bilinear or Hamilton-Adams [2] demosaicking.

The assumption of the method is that while the LL subbands of the red, green, and blue channels are different, their detail subbands are approximately the same. This assumption is accurate in smooth regions and regions with achromatic texture where the color is uniform. 
Before beginning the main iteration, the green channel is first enhanced. The red and green channels are downsampled to keep only the pixels where the red component is known. These downsampled images are decomposed using the redundant wavelet transform (RWT), and the high frequency subbands LH, HL, HH of the green channel are replaced with those from the red channel. The downsampled green channel is reconstructed and inserted in the corresponding locations in the initial green channel demosaicking. This process is done similarly at blue pixel locations with the blue channel.

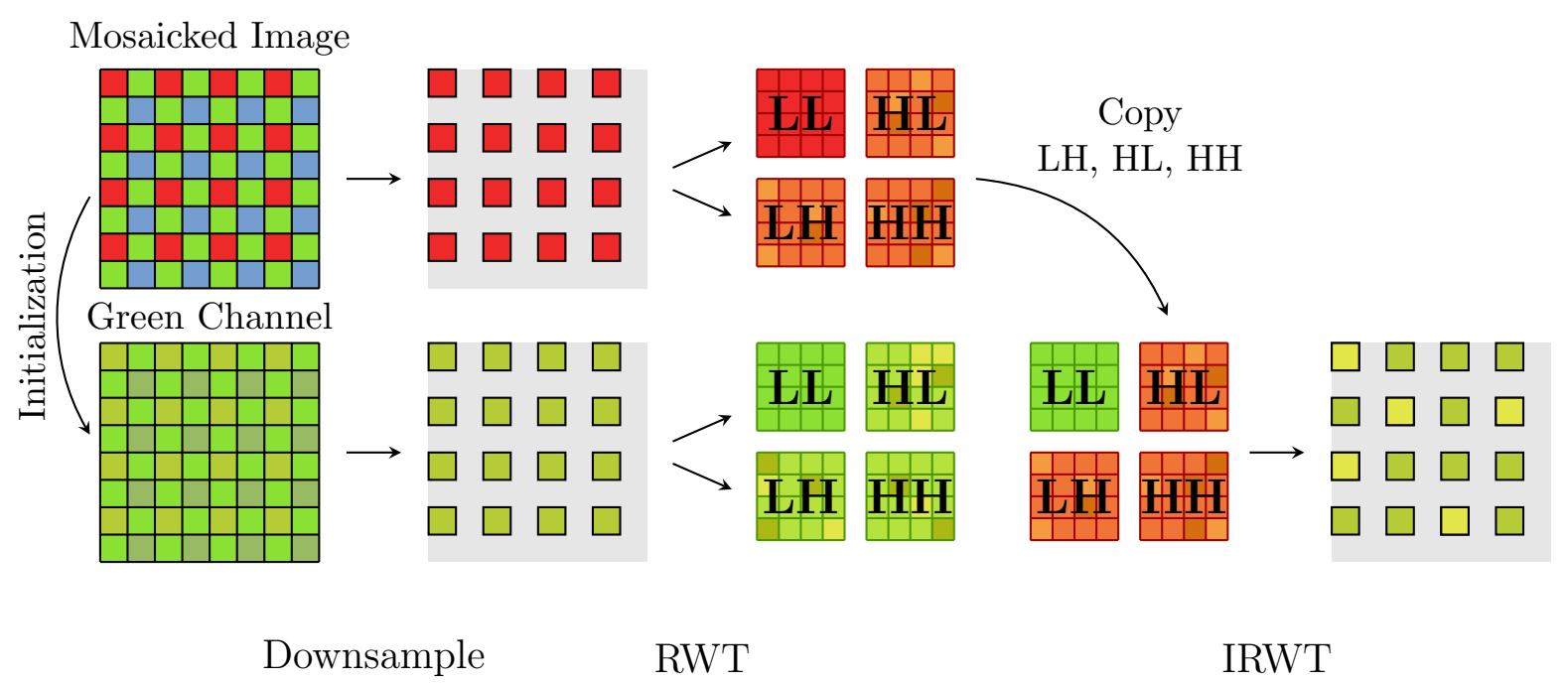

Updating green values at red pixel locations.

The updated green channel is the final estimate of the green channel; it will not change after this point of the algorithm. The main alternating projections iteration begins to estimate the red and blue channels.

1. The first projection copies wavelet details from the green channel to the red and blue channels. The channels are decomposed with the RWT, and the LH, HL, and HH subbands of red and blue channels are replaced with those of the green channel. The channels are then reconstructed.

2. The second projection enforces that the demosaicked result agrees with the observed mosaicked image. The red component at red pixel locations and the blue component at blue pixel locations are set to the known values. The green components at green pixel locations already agree with the mosaicked image, so these do not need to be corrected. 


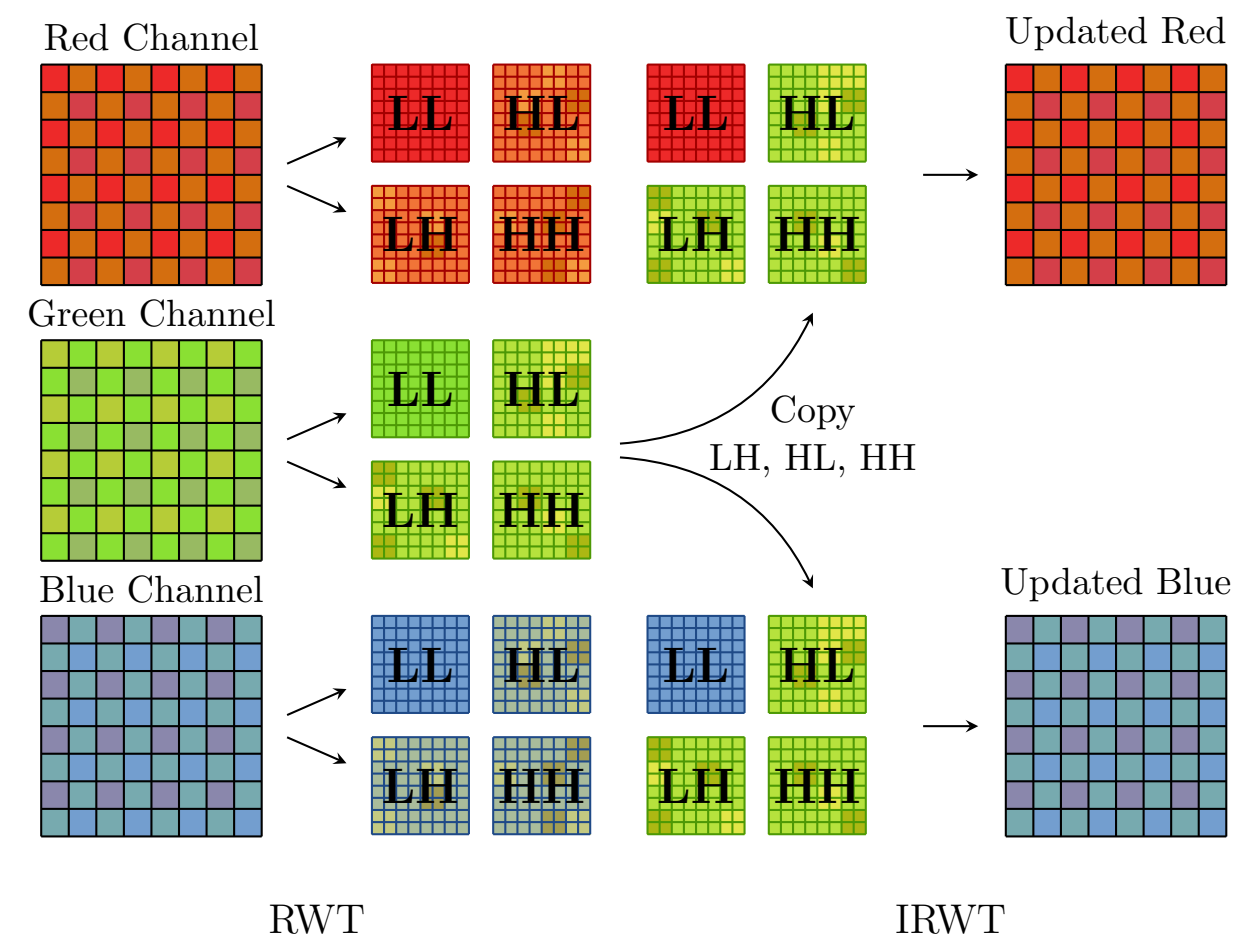

Copying wavelet details from the green channel to the red and blue channels.

Gunturk et al. suggest the following for the algorithm details:

- Use Hamilton-Adams [2] to perform the initial green channel demosaicking.

- For the redundant wavelet transforms, use the decomposition filters $H_{0}(z)=\left(z+2+z^{-1}\right) / 4$ and $H_{1}(z)=\left(z-2+z^{-1}\right) / 4$ and reconstruction filters $G_{0}(z)=\left(-1 z^{2}+2 z+6+2 z^{-1}-z^{-2}\right) / 8$ and $G_{1}(z)=\left(1 z^{2}+2 z-6+2 z^{-1}+z^{-2}\right) / 8$.

- Perform 8 iterations of the alternating projections loop.

\section{Examples}

The method is quite successful in suppressing color artifacts. This example is on a mostly gray image of a sail. This image is useful to test to what degree the methods create false colors. 
Exact

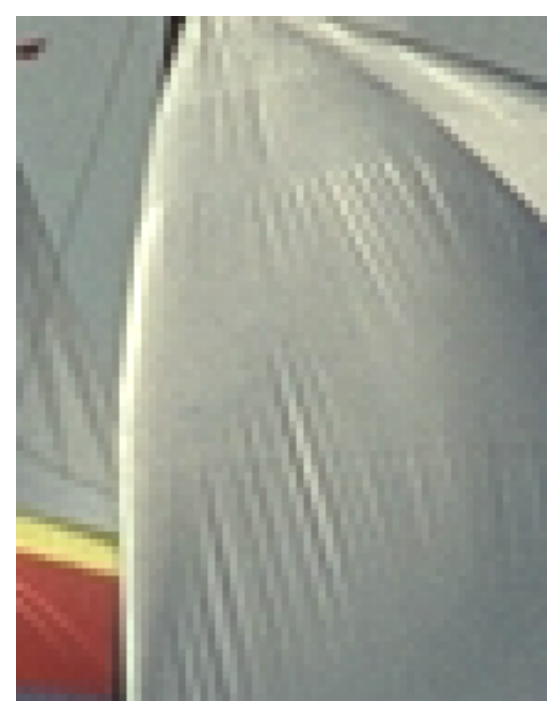

Hamilton-Adams [2] (PSNR 37.45)

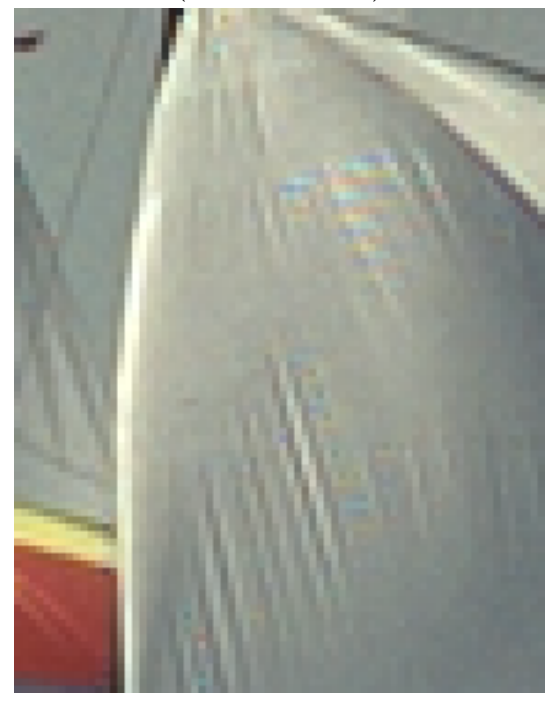

Observed Image

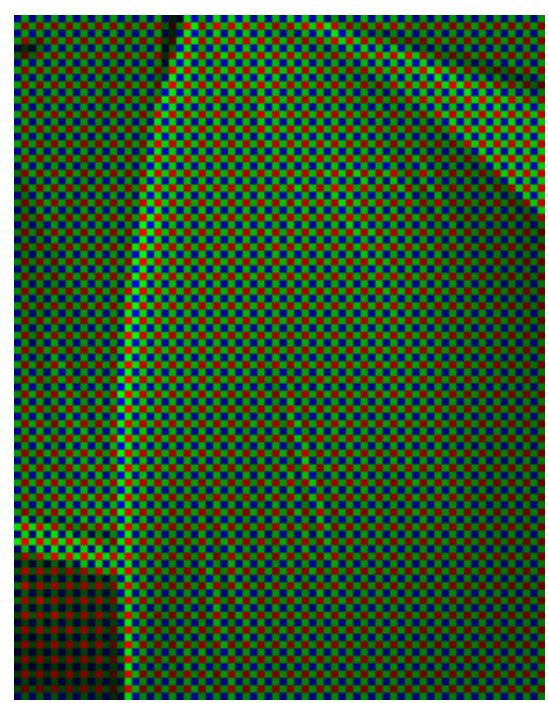

Zhang-Wu [4]

(PSNR 42.71)

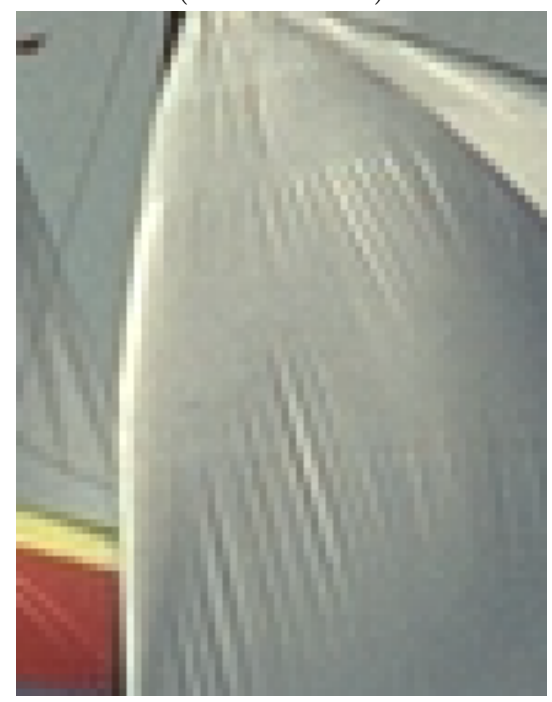

Bilinear

(PSNR 29.91)

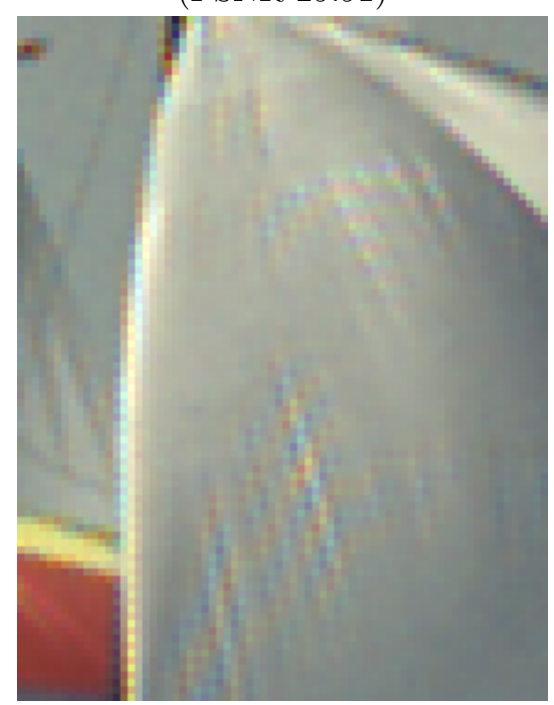

Gunturk et al. (PSNR 40.95)

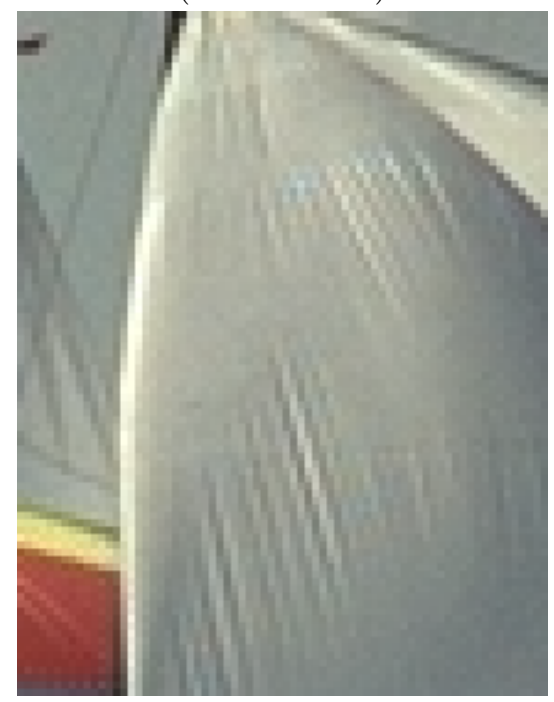


A weakness of the method is that it has a tendency to produce zipper artifacts, unnatural oscillations of bright and dark pixels. This effect is noticeable when the image has significant color discontinuities.

Exact

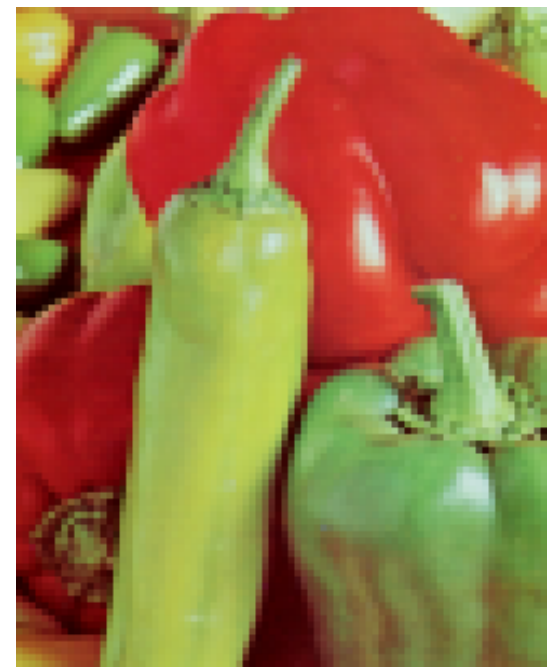

Hamilton-Adams [2] (PSNR 29.17)

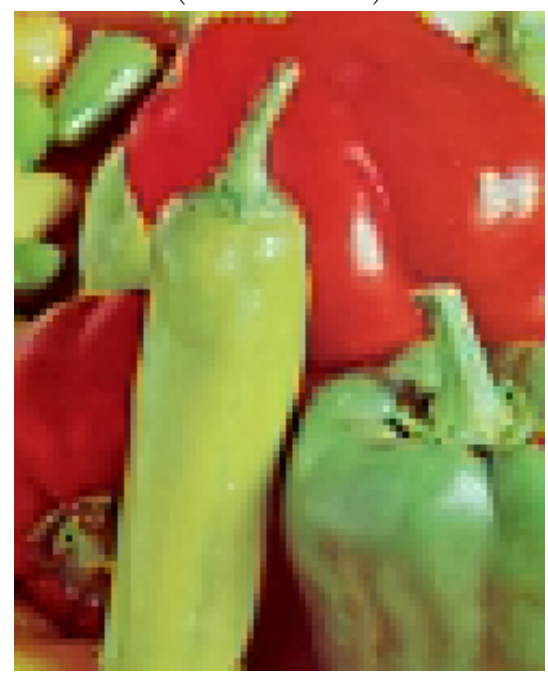

Observed Image

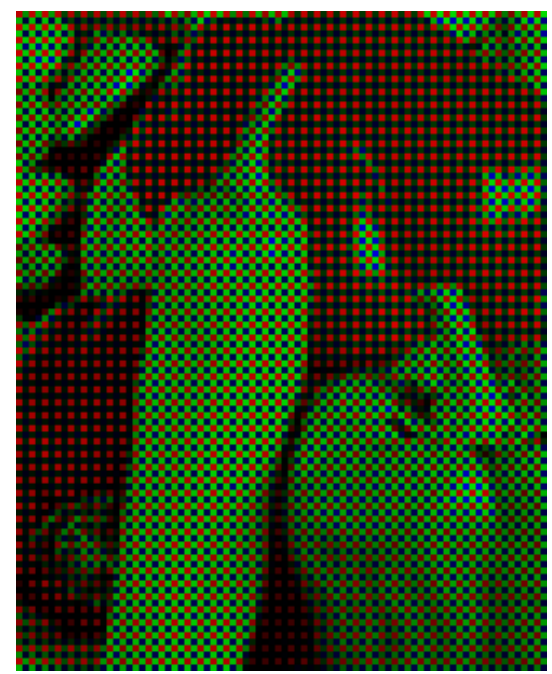

Zhang-Wu [4]

(PSNR 28.94)

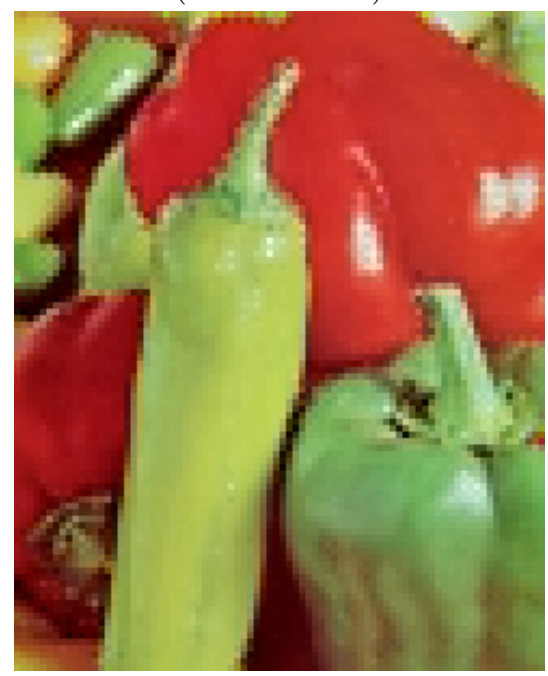

Bilinear

(PSNR 29.47)

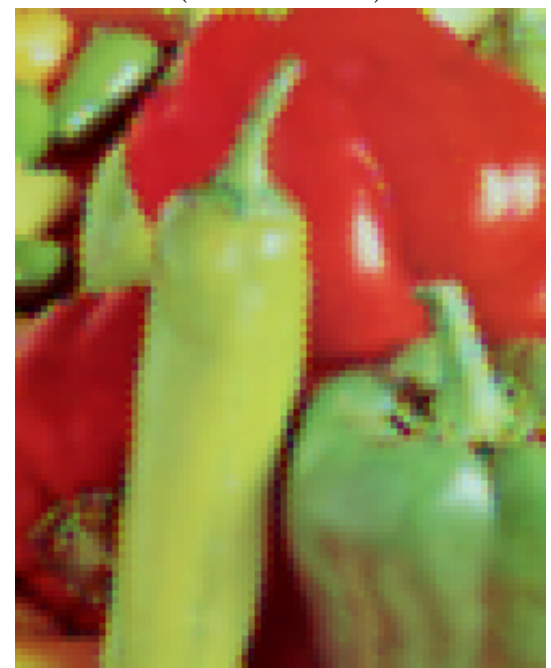

Gunturk et al.

(PSNR 26.98)

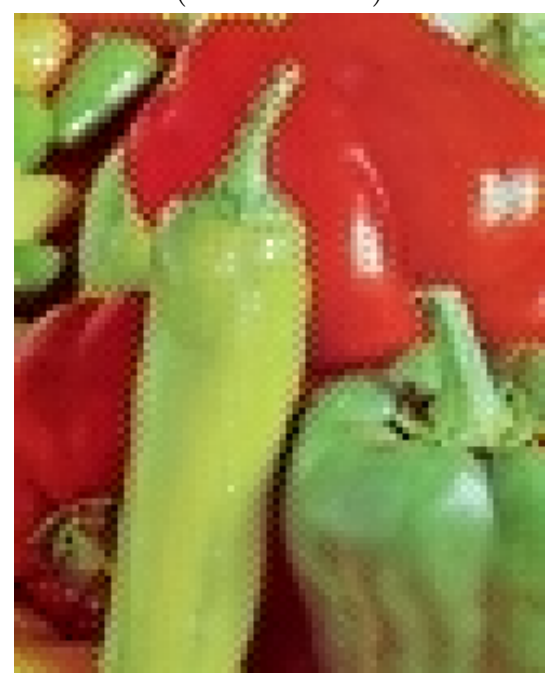

The Gunturk et al. alternating projections method is generally quite resistant to color artifacts. Nevertheless, it is still possible to observe color artifacts when the exact image has fine oscillating patterns. 
Exact
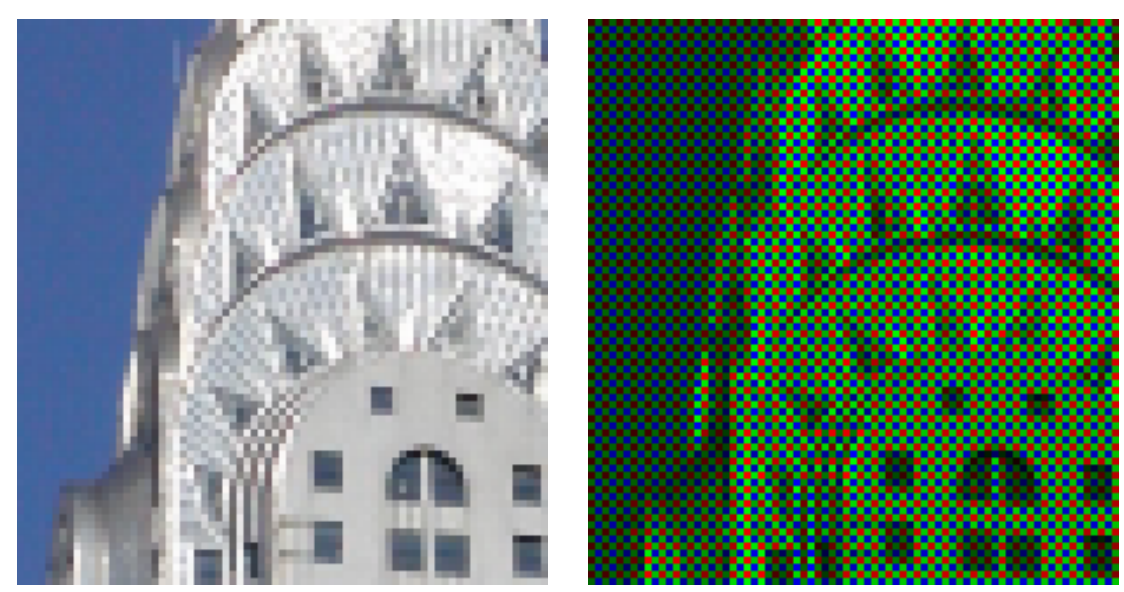

Observed Image

Zhang-Wu [4]

(PSNR 31.09)

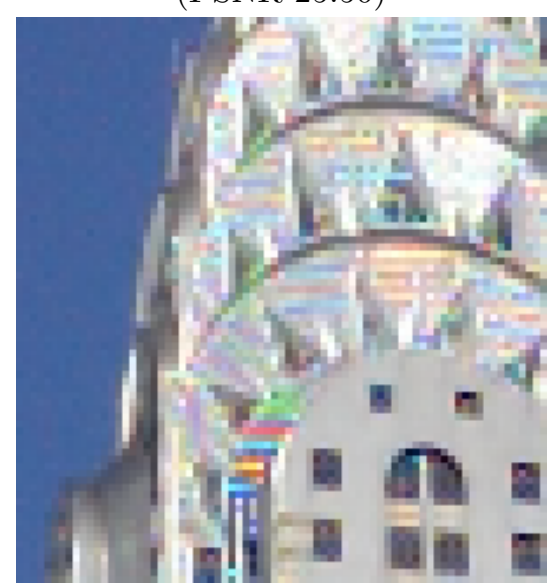

Hamilton-Adams [2]

(PSNR 25.50)

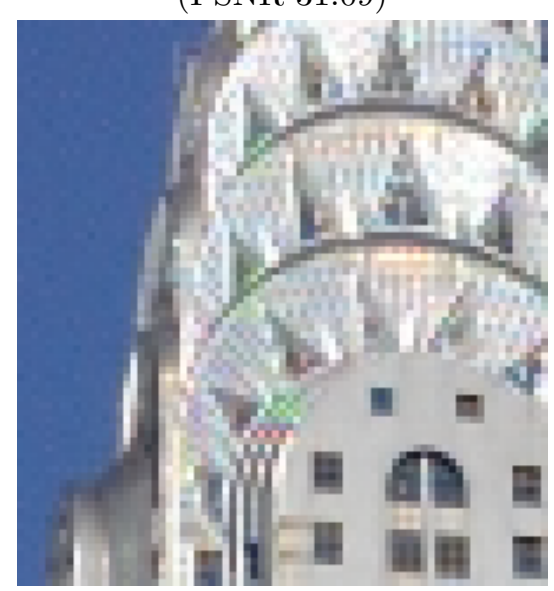

Bilinear

(PSNR 22.15)

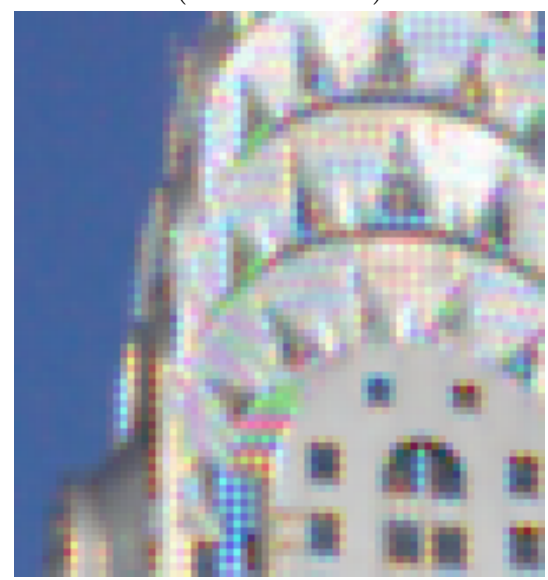

Gunturk et al.

(PSNR 29.59)

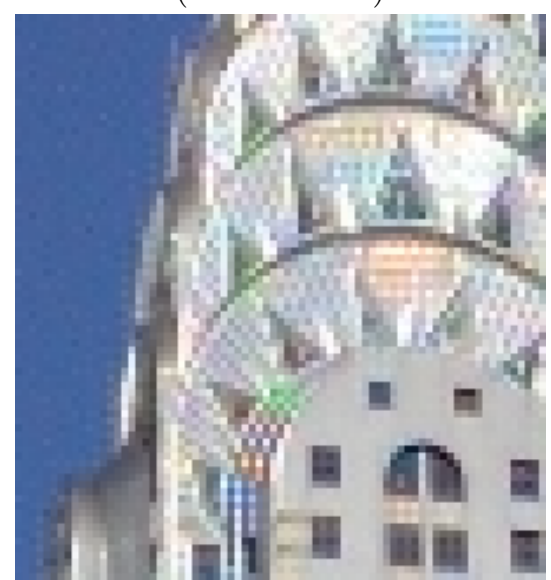

\section{Acknowledgments}

This material is based upon work supported by the National Science Foundation under Award No. DMS-1004694. Work partially supported by the Office of Naval Research under grant N0001497-1-0839 and by the European Research Council, advanced grant "Twelve labours."

\section{Image Credits}

(19)

Kodak Image Suite, image 7 (http://r0k.us/graphics/kodak/)

Kodak Image Suite, image 9 (http://rok.us/graphics/kodak/)

Peppers standard test image

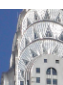

ini Leena Hietanen, CC-BY-SA (http://commons.wikimedia.org/wiki/File:Chrysler_building-_top. jpg) 


\section{References}

[1] B. E. Bayer, "Color imaging array," U.S. Patent 3971065, 1976.

[2] J. F. Hamilton, Jr. and J. E. Adams, Jr., "Adaptive color plan interpolation in single sensor color electronic camera," U.S. Patent 5629734, 1997.

[3] B. K. Gunturk, Y. Altunbasak, and R. M. Mersereau, "Color plane interpolation using alternating projections," IEEE Transactions on Image Processing, vol. 11, no. 9, pp. 997-1013, 2002. http: //dx.doi.org/10.1109/TIP.2002.801121

[4] L. Zhang and $\mathrm{X}$. Wu, "Color demosaicking via directional linear minimum mean square-error estimation," IEEE Transactions on Image Processing, vol. 14, no. 12, pp. 2167-2178, 2005. http: //dx.doi.org/10.1109/TIP.2005.857260 